\title{
Independent Premotor Encoding of the Sequence and Structure of Birdsong in Avian Cortex
}

\author{
Mark J. Basista, ${ }^{1,2}$ Kevin C. Elliott, ${ }^{1,2}$ Wei Wu, ${ }^{1,3}$ Richard L. Hyson, ${ }^{1,2}$ Richard Bertram, ${ }^{1,4}$ and Frank Johnson ${ }^{1,2}$ \\ ${ }^{1}$ Program in Neuroscience and Departments of ${ }^{2}$ Psychology, ${ }^{3}$ Statistics, and ${ }^{4}$ Mathematics, Florida State University, Tallahassee, Florida 32306
}

How the brain coordinates rapid sequences of learned behavior, such as human speech, remains a fundamental problem in neuroscience. Birdsong is a model of such behavior, which is learned and controlled by a neural circuit that spans avian cortex, basal ganglia, and thalamus. The songs of adult male zebra finches (Taeniopygia guttata), produced as rapid sequences of vocal gestures (syllables), are encoded by the cortical premotor region HVC (proper name). While the motor encoding of song within HVC has traditionally been viewed as unitary and distributed, we used an ablation technique to ask whether the sequence and structure of song are processed independently within HVC. Results revealed a functional topography across the medial-lateral axis of HVC. Bilateral ablation of medial HVC induced a positive disruption of song (increase in atypical syllable sequences), whereas bilateral ablation of lateral HVC induced a negative disruption (omission of individual syllables). Bilateral ablation of central HVC either had no effect on song or induced syllable omission, similar to lateral HVC ablation. We then investigated HVC connectivity and found parallel afferent and efferent pathways that transit medial and lateral HVC and converge at vocal motor cortex. In light of recent evidence that syntactic and lexical components of human speech are processed independently by neighboring regions of cortex (Menenti et al., 2012), our demonstration of anatomically distinct pathways that differentially process the sequence and structure of birdsong in parallel suggests that the vertebrate brain relies on a common approach to encode rapid sequences of vocal gestures.

Key words: ablation; motor encoding; parallel processing; serial-order behavior; tract-tracing; zebra finch

\section{Introduction}

Like human speech, birdsong requires precise sequencing of learned vocal gestures. In zebra finches, song is encoded by the cortical premotor region HVC (Long and Fee, 2008; Goldin et al., 2013) (Fig. 1), but little is known about how neural activity at any given HVC location contributes to the song pattern as a whole. In humans, distinct yet slightly overlapping regions of cortex encode syntactical and lexical components of speech independently (Menenti et al., 2012), leading us to ask whether a similar parallel architecture might underlie neural encoding of the sequence and structure of birdsong.

Whereas earlier evidence suggested unitary encoding of song sequence and structure within HVC (for review, see Margoliash et al., 1994), recent findings raise the possibility of a functional topography in the motor encoding of song. For example, HVC is organized by connectivity and physiology into multiple rostralcaudal swaths or chains of neurons with limited medial-lateral connectivity between them (Stauffer et al., 2012) (diagrammed

Received May 12, 2014; revised Oct. 31, 2014; accepted Nov. 3, 2014.

Author contributions:M.J.B., K.C.E., R.L.H., R.B., and F.J. designed research; M.J.B. and K.C.E. performed research; M.J.B., K.C.E., W.W., and F.J. analyzed data; M.J.B., K.C.E., and F.J. wrote the paper.

This work was supported by National Science Foundation Grant IOS 1146607. We thank Dr. Ofer Tchernichovski for use of the Sound Analysis Pro software and the two anonymous reviewers who made numerous suggestions that improved the manuscript.

The authors declare no competing financial interests.

Correspondence should be addressed to Dr. Frank Johnson, Program in Neuroscience and Department of Psychology, Florida State University, Tallahassee, FL 32306-4301. E-mail: johnson@psy.fsu.edu.

DOI:10.1523/JNEUROSCI.1940-14.2014

Copyright $\odot 2014$ the authors $\quad 0270-6474 / 14 / 3416821-14 \$ 15.00 / 0$ over left HVC in Fig. 1B). Indeed, excitatory neural activity traverses the rostral-caudal axis of HVC in vivo (Day et al., 2013), and medial and lateral halves of HVC can be physically disconnected from each other without altering the song pattern (Poole et al., 2012). Together, these data suggest the hypothesis that medial and lateral HVCs are capable of functioning independently and could encode different dimensions of the vocal pattern in parallel.

Birdsong is under dual premotor control; that is, both HVC and the lateral magnocellular nucleus of the anterior nidopallium (LMAN) convey premotor signals to the primary motor cortical region for song, the robust nucleus of the arcopallium (RA) (see Fig. 1A) (for review, see Bertram et al., 2014). Whereas HVC premotor activity encodes the timing of song (Long and Fee, 2008; Goldin et al., 2013), LMAN premotor activity contributes adaptive variability to the vocal-motor stream (Kao and Brainard, 2006; Aronov et al., 2008). Because weakening one premotor stream shifts the character of vocal production in favor of the other (Thompson et al., 2007, 2011), we explored the premotor encoding of song in HVC in the absence of LMAN activity.

HVC is located near the dorsal surface of the avian telencephalon, making it accessible to precise, visually guided surgical manipulation (see Fig. 1B). Here we report that bilateral ablation within medial HVC induces a positive disruption of song (increase in atypical syllable sequences), whereas bilateral ablation within lateral HVC induces a negative disruption (omission of one or more syllables). Ablation within central HVC either produces no deficit (compare Poole et al., 2012) or the omission of individual song syllables. We then used tract-tracing techniques 
to demonstrate that the extrinsic connectivity of medial and lateral HVC is organized in parallel. The observed functional topography of HVC (medial and lateral HVC differentially process the sequence and structure of birdsong in parallel) suggests that human and avian brains rely on a common architecture to encode complex sequences of vocal gestures.

\section{Materials and Methods}

Animals and environment

Male zebra finches $(N=34)$ were raised in our breeding colony to adulthood ( $>120 \mathrm{~d}$ after hatch), after which they were transferred to individual cages in sound-attenuating chambers that also provided visual isolation. These chambers were equipped with microphones to capture vocal behavior throughout the experiment. Food and water were provided ad libitum for the duration of the experiment. The birds were maintained on a $14 \mathrm{~h}$ light $/ 10 \mathrm{~h}$ dark photoperiod. At least 1 week was allowed for the birds to acclimate to the recording environment before singing was collected and analyzed. We selected 1 week based on behavioral observations that adult male zebra finches achieve a stable baseline of daily song bout production after 1 week of housing in the sound-recording chambers (F.J., unpublished observations). All daily care and experimental procedures of the birds were reviewed and approved by the Florida State University Animal Care and Use Committee.

Experimental and control surgeries

After the acclimation period, and between $3 \mathrm{~d}$ and 1 week of preoperative song recording, birds underwent a surgical procedure that combined the following: (1) retrograde labeling of HVC neurons that project to Area X (for postmortem visualization and measurement of HVC), (2) bilateral ablation of LMAN, and (3) bilateral ablation of medial (MED), lateral (LAT), or central (CENT) HVC. Song production is under dual premotor control (Fig. 1A) (Bertram et al., 2014), and LMAN ablation is necessary to isolate HVC premotor drive on song (Kao and Brainard, 2006; Thompson and Johnson, 2007; Thompson et al., 2007, 2011). The control group consisted of birds that received bilateral ablation of tissue immediately surrounding but not including HVC (CTL). In some birds that received bilateral ablation of $\mathrm{HVC}$ subregions, LMAN remained intact (whole or in part, see Fig. 2), and these birds were designated as a separate group (LMANR). Consequently, groups were $\operatorname{MED}(N=5)$, LAT $(N=5)$, CENT $(N=6)$, CTL $(N=5)$, and LMANR $(N=6)$.

Before surgery, each bird was deeply anesthetized with Equithesin $(0.04 \mathrm{ml})$ and secured in a stereotaxic instrument. The scalp was incised down the midline of the head, exposing the skull. The skull surface directly above the bifurcation of the midsagittal sinus defined stereotaxic zero, and a head angle of $24^{\circ}$ was set between stereotaxic zero and a skull surface point located $5 \mathrm{~mm}$ anterior from stereotaxic zero. Craniotomies over left and right HVC as well as left and right AreaX/LMAN were made using predetermined coordinates (see below).

\section{A Songbird Vocal Control Network (abridged)}
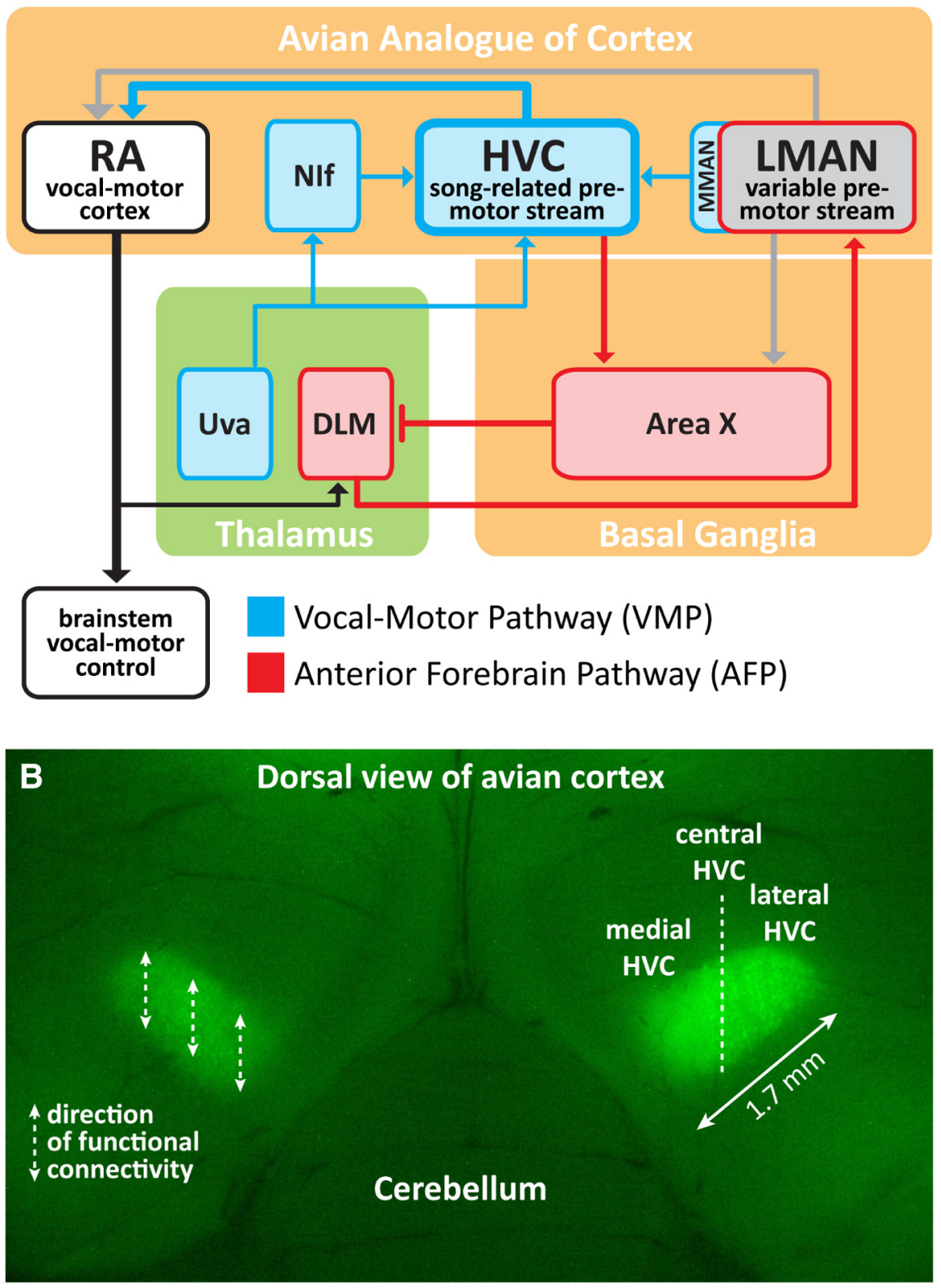

Figure 1. Sagittal schematic of the zebra finch vocal control network and an image of HVC (proper name), the premotor cortical region under study. $\boldsymbol{A}$, The learned song of adult birds is an integration of dual premotor streams: HVC encodes the temporal structure of adult song (Long and Fee, 2008; Goldin et al., 2013), whereas LMAN contributes adaptive variability into the vocalmotor stream that is important during juvenile learning (Aronov et al., 2008; Elliott et al., 2014) and in adulthood (Kao and Brainard, 2006; Thompson et al., 2011). Weakening of one premotor stream shifts the character of vocal production in favor of the other (Thompson et al., 2007, 2011). For this reason, LMAN was ablated in all birds (unless otherwise specified) to isolate HVC premotor drive on song. $\boldsymbol{B}$, The dorsal location of HVC is ideal for experimental manipulation; left and right HVC visualized via retrograde transport of DiO. Dashed lines over left HVC indicate the rostral-caudal functional organization of HVC (Stauffer et al., 2012). Because the motor encoding of song is distributed across left and right HVC (Ashmore et al., 2008; Long and Fee, 2008; Wang et al., 2008), all reported findings are the result of bilateral manipulations to medial, central, or lateral HVC.

Retrograde labeling of HVC neurons. HVC neurons that project to Area $\mathrm{X}$ were retrogradely labeled with a vital fluorescent dye (DiO or DiI, 5\% in DMSO, Invitrogen) to unambiguously identify the borders of HVC during postmortem validation of the location and extent of ablations targeted to one of three HVC subregions (MED, LAT, CENT; see below). For this study, the injection of vital dyes into Area $\mathrm{X}$ alone is without effect on the structure of adult song (M.J.B., unpublished observations). Glass micropipettes (World Precision Instruments, 1.2 outer diameter/ 0.68 inner diameter) were pulled using a vertical pipette puller (David 
A

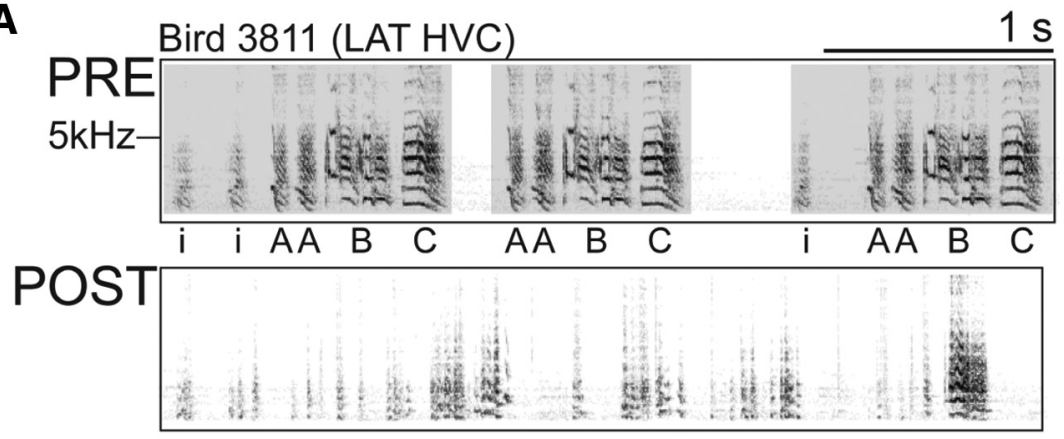

B

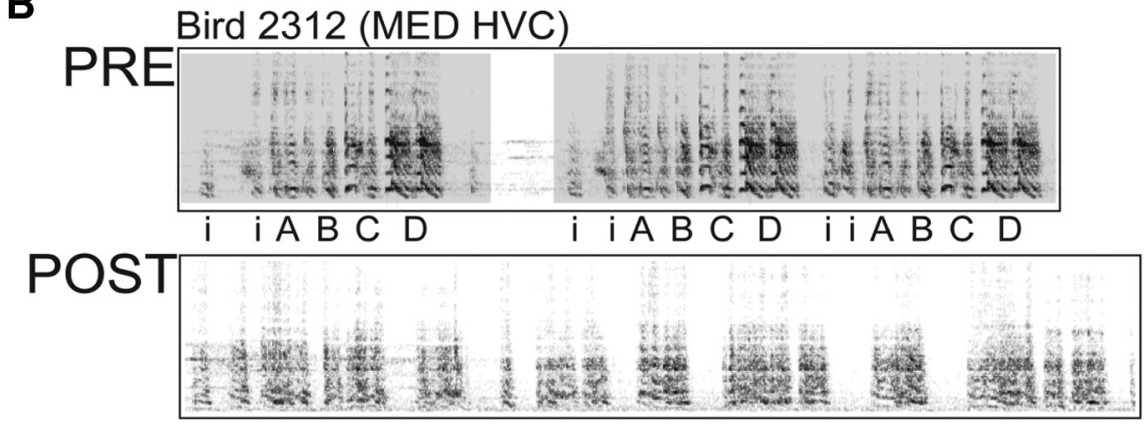

Figure 2. In the presence of LMAN, bilateral HVC damage produces a complete loss of song structure and sequence. Representative PRE and POST spectrograms from LMANR birds that received bilateral damage to lateral ( $\boldsymbol{A}$ ) or medial (B) HVC, but not LMAN ablation.

Kopf Instruments, model 720 ) and used to inject $\sim 100$ nl of dye into Area $\mathrm{X}$ with a nitrogen gas-pressure system (Applied Scientific Instruments, MPPI-3). Dye injections targeted Area X bilaterally using coordinates 3.5 $\mathrm{mm}$ anterior of stereotaxic zero, $1.5 \mathrm{~mm}$ lateral of the midsagittal sinus, and $4 \mathrm{~mm}$ ventral from the surface of the brain.

Bilateral ablation of LMAN. To bilaterally ablate LMAN, a Tefloninsulated tungsten electrode was placed at bilateral sites $1.3 \mathrm{~mm}$ and 1.8 $\mathrm{mm}$ lateral of the midsagittal sinus, $3.7 \mathrm{~mm}$ anterior to stereotaxic zero, and $2.7 \mathrm{~mm}$ ventral from the surface of the brain; $100 \mu \mathrm{A}$ of current was passed through the electrode for $3 \mathrm{~min}$ at each ablation site. The location and extent of bilateral LMAN ablations were validated postmortem using Nissl-stained tissue sections (see below).

Bilateral ablation of MED, LAT, or CENT HVC. Located at the dorsal surface of avian cortex (Fig. 1B), HVC is comprised of three cell populations, excitatory RA-projecting neurons (HVCRA), excitatory Area $\mathrm{X}$-projecting neurons (HVCX), and inhibitory interneurons (HVCINT), arranged in a superficially isomorphic mosaic (Fortune and Margoliash, 1995; Wild et al., 2005). HVC also contains no known fibers of passage. However, the synaptic connectivity and physiology of HVC exhibit a clear axial organization. HVC neurons form a parallel series of interconnected rostral-caudal swaths that show limited medial-lateral communication (Fig. 1B, left) (Stauffer et al., 2012). Therefore, we used an electrolytic ablation technique to provide a greater level of surgical control over the location, completeness, and spatial extent of ablations. Bilateral ablations targeting MED, LAT, or CENT HVC were visually guided following resection of the dura mater over HVC. When viewed from a dorsal perspective through a surgical microscope, the use of indirect lighting revealed HVC as an oblong white mass (due to heavy myelination) with white axon bundles extending from the rostral and caudal edges, distinguishing HVC from the less myelinated brain tissue that surrounds it.

LAT and MED HVC ablations were made by lowering the tungsten electrode to a depth of $0.4 \mathrm{~mm}$ from the surface of the brain and applying $100 \mu \mathrm{A}$ of current. By varying the number of ablation sites and current durations, we customized the ablation approach for each bird based on the observed size and shape of HVC, which is known to vary twofold in volume across individual birds (MacDougall-Shackleton et al., 1998; Airey and DeVoogd, 2000). We used either bilaterally mirrored sites consisting of $6-8$ locations ( $15 \mathrm{~s}$ of current per location) separated by $0.3 \mathrm{~mm}$ and arranged in a grid that encompassed the medial or lateral portion of HVC, or we used bilaterally mirrored sites at 2 locations ( $60 \mathrm{~s}$ per location) that were separated by $0.3 \mathrm{~mm}$ along the rostralcaudal axis of medial or lateral portions of HVC. Bilateral ablation of CENT HVC was accomplished by placing an electrode at a series of 3-4 sites ( $15 \mathrm{~s}$ of current per location) separated by $0.3 \mathrm{~mm}$ along the rostral-caudal axis of central HVC. Although our visually guided approach ensured that ablations were customized to the size and shape of each bird's HVC (and were specifically targeted to MED, LAT, or CENT HVC), we validated the location and extent of the ablations via postmortem quantification of HVC volume in serial sections, with the borders of HVC defined by the distribution of retrogradely labeled Area X-projecting HVC neurons.

HVC CTL surgeries. Once HVC was exposed as described above, the ablation electrode was placed outside the medial, lateral, or ventral borders of HVC at bilaterally mirrored sites. These sites were located visually or by penetrating below the ventral border of HVC $(>0.6 \mathrm{~mm}$ below the surface of the brain); $100 \mu \mathrm{A}$ of current was delivered for $60 \mathrm{~s}$ at each ablation site.

\section{Ablation validation}

Tissue collection. At the end of a 2 week postoperative recording period, all birds were killed using an overdose of Equithesin $(0.08 \mathrm{ml})$ and perfused intracardially with PBS, followed by ice-cold $4 \%$ PFA to fix the brain tissue. Brains were dissected from the skull and postfixed in PFA overnight. Brains were then sectioned in the sagittal plane using a vibratome (Leica, VT1000S). In birds with HVC subregion ablation (MED, CENT, or LAT HVC birds), section thickness was set to $60 \mu \mathrm{m}$ to facilitate discrimination between intact and ablated regions of HVC during serial section reconstruction. Section thickness was set to $120 \mu \mathrm{m}$ in birds where HVC remained intact (HVC CTL birds). Serial sections containing LMAN ablation sites were mounted onto microscope slides, Nissl-stained with thionin, defatted with xylene, and coverslipped (Gerfen, 2003). Serial sections containing fluorescently labeled HVC were mounted on microscope slides and coverslipped with saline and then imaged under epifluorescence. These sections were subsequently Nissl-stained, defatted, and coverslipped (Gerfen, 2003), as some of these sections also contained the LMAN ablation sites.

Imaging and reconstruction. Tissue sections were imaged serially using epifluorescent or bright field illumination under a microscope (Leica, DM5000B) interfaced with a PC computer and digital camera (Optronics, MicroFire). Captured images were used to construct quantitative $3 \mathrm{D}$ models of the location and extent of HVC ablation sites using Neurolucida software (Microbrightfield). In each section, the distribution of fluorescently labeled cells was used to define the borders of HVC as well as the presence of any autofluorescent ablation-damaged tissue (see representative tissue sections in Fig. 3). Tracings excluded paraHVC, which is located medial to HVC and projects to Area X but not to RA. In sagittal sections, paraHVC lacks the characteristic oval shape of HVC and appears as a thin strip of cells oriented along the dorsoventral extent of the caudal-most portion of the telencephalon (Foster and Bottjer, 1998; Olson et al., 2011). Ablation damage to paraHVC occurred in some (3 of 5) birds with MED HVC ablations, but birds with control ablations that included only paraHVC were not disrupted in their postoperative singing and were included in the CTL group.

For each bird, estimates of the unilateral volumes of the left and right HVC ablations were determined using the dimensions of the 3D reconstructions. LMAN ablation was confirmed by visually inspecting the ablation site and surrounding sections. In cases where portions of LMAN 
were found to remain, the volume was estimated in the same manner as HVC volume and divided by an average unilateral LMAN volume of $0.11 \mathrm{~mm}^{3}$ to determine the percentage LMAN remaining (compare Thompson and Johnson, 2007).

\section{Song recording and analysis of} acoustic structure

Song recording. Singing was monitored continuously by overhead microphones interfaced with a PC running sound-triggered recording software (Avisoft Recorder, Avisoft Bioacoustics) sampling at $44 \mathrm{kHz}$ before and after surgery. Three days of preoperative singing (PRE) were recorded to ensure that birds were singing stable adult songs. After surgery, the birds were allowed to recover in their home cages for 2 weeks, during which time all postoperative singing (POST) was recorded. All analyzed singing was undirected (i.e., not elicited by the presence of a female).

Analysis of syllable acoustic features. The zebra finches in this study produced songs that consisted of several (range of 2-7) discrete vocalizations (syllables, defined by continuous amplitude envelopes that were separated from one another by small gaps of silence), produced in a canonical sequence (the motif). The motif is sung either once or several times to form a bout of singing. The first 100 song bouts on each of the last $3 \mathrm{~d}$ before surgery (PRE1, PRE2, and PRE3) were analyzed using the Feature Batch tool in Sound Analysis Pro (SAP) software (Tchernichovski et al., 2000). Amplitude thresholds in SAP were customized to consistently segment all individual syllables in each bird and the duration, pitch, frequency modulation (FM), entropy, and pitch goodness of all song syllables contained in the first 100 song bouts from PRE1-PRE3 were measured.

As anticipated, in the absence of LMAN, the acoustic structure of individual song syllables produced at POST resembled their counterparts at PRE (Thompson and Johnson, 2007; Thompson et al., 2007) and did not change over the 2 week postoperative period. In contrast, the POST songs of birds that received partial HVC ablation with LMAN remaining (the LMANR group) showed little resemblance to their PRE songs (Fig. 2 ), and this also did not change over the 2 week postoperative period.

To capture the stability of POST singing, SAP was used to quantify the acoustic properties of all syllables produced on three selected POST days. The first day analyzed (POST1) was 6-8 d after surgery, when the amount of daily song bout production began to return to PRE levels (Thompson et al., 2007, their Fig. 3) and provided a sufficient corpus of song syllables for statistical comparison to the sequence and structure of PRE singing. The second and third POST days (POST2, POST3) were selected from the second week of POST singing, with POST3 typically being the last (14th) postoperative day. One bird in the LMANR group experienced a postoperative period of 3 weeks to determine whether song would recover, which it did not. For this bird, POST2 and POST3 singing was analyzed over after operative days 13-21. Within-group statistical tests confirmed the stability of POST singing across the three selected days (data not shown), and data were therefore combined for analysis of between-group differences.

For each day of singing (PRE1-PRE3, POST1-POST3), the SAP measures of syllable acoustic properties were exported to Excel (Microsoft) and song syllables were visualized by generating scatterplots of syllable acoustic features. This allowed any nonsong vocalizations (calls) and noises that were present in the song files to be identified and removed. Then, for each bird, scatterplots of pairs of acoustic features (syllable duration vs syllable pitch, syllable duration vs syllable FM, syllable duration vs syllable entropy, syllable duration vs syllable pitch goodness) were generated from each day of singing and compared with a scatterplot of the cognate acoustic feature pair from PRE1. We used the KullbackLeibler (KL) distance (Wu et al., 2008) to quantify differences between the scatterplots. KL distance measures the degree of difference between two probability density distributions (in our case, scatterplots from 2 different days of singing). The procedure for this analysis mirrored Thompson et al. (2007), and the equation used is described in detail by Wu et al. (2008). Briefly, a $15 \times 15$ grid of bins was overlaid on each scatterplot, and the data points (syllables) that fell within each bin were counted. When all bins contained similar numbers of data points from both PRE1 and the comparison day, this resulted in a near-zero KL distance (indicating that the singing from both days was similar). When bins had few or no data points from $1 \mathrm{~d}$, but many from another day, this increased the KL distance (indicating that the distribution of data points from the $2 \mathrm{~d}$ of singing did not overlap). Dissimilarity (or an increase in $\mathrm{KL}$ distance) between days of singing occurs when there is change in the central tendency of the acoustic features of a syllable (e.g., the mean duration and/or pitch of a syllable shifts from $1 \mathrm{~d}$ to another) and/or a change in the dispersion of the acoustic features of a syllable (e.g., a bird sings their syllables with a more or less consistent duration and/or pitch on $1 \mathrm{~d}$ vs another).

Scatterplots from PRE1 were compared with scatterplots from all other PRE (PRE2, PRE3) and POST (POST1-POST3) days, and KL distance values for all four feature pairs were calculated (syllable duration vs syllable pitch, syllable duration vs syllable FM, syllable duration vs syllable entropy, syllable duration vs syllable pitch goodness). With the exception of the LMANR group (where no PRE syllables were identifiable at POST; Fig. 2), we only analyzed syllables produced at both PRE and POST. This controlled for false large KL distance values, which could be generated if a syllable was present at PRE but omitted at POST (as occurred in LAT HVC and some CENT HVC birds). All PRE1 versus PRE2 and PRE3 comparisons were low across all groups (KL values $<1$; data not shown) indicating the expected stability of preoperative song structure. For PRE1 versus POST1-POST3 comparisons, KL distance values were stable within groups and were therefore averaged to create a single "POST" value for each feature pair. We then generated a composite KL 
distance for POST singing by averaging values from the four feature pairs.

\section{Analysis of syllable omission and atypical syllable transitions}

Motif quantification. We used SongSeq software (Daou et al., 2012) to establish the identity and quantify the sequence of the populations of PRE and POST song syllables that were isolated by SAP. The song syllables of each bird were identified (e.g., syllable A, syllable B, etc.) in SongSeq by indicating where different syllable clusters were located on a scatterplot of syllable duration and a selected acoustic feature (e.g., syllable duration vs syllable entropy). For each individual bird, the syllable duration versus syllable feature scatterplot that produced the most distinct clustering of syllables was used: this varied from bird to bird depending on the spectral and temporal properties of each bird's syllables (for additional details, see Daou et al., 2012; Thompson et al., 2011; Wu et al., 2008). Introductory syllables were not included in this analysis as no effect on their production was observed. SongSeq mapped the identity of each syllable into an Excel spreadsheet in the order that each syllable was produced within each recorded song bout.

To quantify whether syllables were omitted from a bird's motif, it was necessary to measure the number of motifs that contained the full PRE syllable repertoire in both PRE and POST singing, and compare them. Because SongSeq logs the sequence of syllables present in each bout of singing into an Excel spreadsheet, it allows simple visual identification of syllable sequences that match each bird's canonical motif. Each motif syllable was assigned a unique identifying color, resulting in repeated color patterns in the data sheet that indicated repeated production of motifs.

To quantify how many motifs were produced during each day of singing, we identified a syllable that was consistently produced only once during each motif. Importantly, we selected a syllable that was produced even when motifs were truncated. It is not uncommon for zebra finches to omit the last syllable (or syllables) of their motif in a portion of their song bouts (this occurred $\sim 30 \%$ of the time in the PRE singing of the birds used here, which we confirmed by comparing data sheets and spectrograms of corresponding sound files). To measure the total number of complete and truncated motifs, syllables from the middle of the motif were selected. However, the first or second syllable was used when birds did not have a consistently produced middle syllable. In this way, the number of times that the selected syllable was produced indexed the number of motifs (complete and truncated) that the bird produced during a given day of singing. The ratio of complete motifs to total motifs for each bird was then calculated as follows:

$\frac{\text { \# of complete motifs }}{\text { Total \# of motifs }}$

$$
=\% \text { of motifs containing full PRE repertoire }
$$

Syllable production analysis. For each bird, the color-coded pattern in the SongSeq data sheet also revealed one or more syllables that were produced only when the complete motif was produced. Syllables at the end of the motif were targeted by this procedure. The number of instances of this syllable indexed the total number of complete motifs during a day of singing. Again, this was confirmed by comparing data sheets and spectrograms of corresponding sound files. The number of complete motifs was then divided by the total number of complete and truncated motifs, which represented the percentage of motifs that contained the full PRE repertoire. If a bird stopped singing its full PRE repertoire of syllables entirely at POST (as determined by the absence of any measured instances of one or more motif syllables), then a 0 was assigned to the dividend of the equation, resulting in a quotient of 0 , and an estimation of $0 \%$ of motifs containing the full PRE repertoire of syllables.

Analysis of the positions of omitted syllables within motifs. When syllables were omitted at POST, we also asked whether syllable omission was more likely to occur at the beginning, middle, or end of motifs. The zebra finches in this study had songs that ranged from 2 to 7 unique syllables, and all birds, except for one bird in the CTL group, had 3 or more syllables in their song. In birds with 4 or more syllables in their song, it was necessary to collapse the syllables in the middle of the song into one category (middle) so that comparisons could be made between birds. To do this, the first and last syllables were quantified separately, and any syllables in between were classified as "middle" (e.g., for the motif A-BC-D, syllable A would be classified as the first syllable, D as last syllable, and B and C middle syllables). The CTL bird that sang only 2 syllables was classified as having only a first syllable and a last syllable. For each day, the number of instances of each syllable was divided by the total number of complete and truncated motifs:

$$
\frac{\# \text { of each } \text { syllable }_{i}}{\text { Total \# of motifs }}=\text { syllable }_{i} \text { per motif }
$$

where $i=$ first, middle, or last syllables.

This estimated the number of times a syllable was produced per motif per day. This number was then summed across POST days and divided by the sum from all PRE days as follows:

$$
\frac{\text { \# of POST syllables }}{\text { p per motif }}
$$

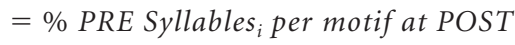

This quotient represented the percentage change between PRE and POST of the likelihood of a first, middle, or last syllable being produced during a motif.

Syllable transition analysis. SongSeq also quantified how many instances of different syllable transitions were present during each day of singing. To ensure similar measurement across all experimental groups, only transitions that could not be explained by syllable omissions or motif truncation were quantified. That is, to accommodate the omission of syllables by LAT HVC (and some CENT HVC) birds, we counted only transitions from song bouts that contained all of the syllables that a bird sang at POST. Transitions were then classified as either typical or atypical. Typical transitions were defined as the set of syllable transitions present within each bird's canonical motif at POST. We excluded intermotif transitions (e.g., when a bird sang two motifs in a row, A-B-C-D, A-B-C-D, the D-A transition was not measured). Conversely, any transitions not present within each bird's canonical motif at POST were classified as atypical. Atypical transitions were summed across all $3 \mathrm{~d}$ of PRE singing and then divided by the sum of all transitions to determine what percentage of transitions were atypical at PRE as follows:

$$
\frac{\# \text { of atyptical transitions }}{\text { Total \# of transitions }}=\% \text { of transitions that are atypical }
$$

This process was then repeated for the $3 \mathrm{~d}$ of POST singing, revealing the percentage of atypical transitions at PRE and POST.

Analysis of the positions of syllables that preceded atypical transitions. When atypical transitions occurred at POST, we also asked whether the position of a syllable within the motif influenced the likelihood that it would be followed by an atypical transition. Again, syllables were classified as first, middle, or last. All atypical transitions that began with the first syllable were summed across all 3 POST days and were divided by the sum of all transitions to determine the percentage of atypical transitions that began with the first syllable as follows:

$$
\begin{aligned}
& \frac{\text { \# of transitions beginning with syllable }}{i} \\
& \begin{aligned}
\text { total \# of transitions } \\
\quad=\% \text { atypical transitions that follow syllable }
\end{aligned}
\end{aligned}
$$

where $i=$ first, middle, or last syllables.

This was repeated for middle and last syllables for each bird. This procedure revealed the position of syllables that were most likely to precede an atypical transition at POST.

\section{Extrinsic connectivity of $H V C$}

Injections of bidirectional tract-tracing dyes into HVC. Behavioral results from birds with MED and LAT HVC ablations showed a partitioning of function across medial and lateral HVC, suggesting that the extrinsic connectivity of HVC might be organized into parallel medial and lateral 
streams. In a separate group of birds $(N=7)$, focal pressure injections of two retrograde/anterograde tracers ( $\mathrm{DiI}$ or $\mathrm{DiO}, 5 \%$ in $\mathrm{DMSO}$ ) were made at two different locations within the mediolateral axis of HVC. The distance between DiI and DiO injection sites was varied (range: $0.1-1$ $\mathrm{mm}$ ) to demonstrate experimental control over double- versus singlelabeling of neurons in regions that are afferent to HVC. To ensure the precise targeting of tracer injections, HVC was visualized as described above for the HVC ablation surgeries. Tracers were delivered into HVC ( $\leq 40 \mathrm{nl}$ ) using glass micropipettes as described above. Tracers were delivered at a depth of $0.45 \mathrm{~mm}$ from the surface of the brain.

Tissue collection. One week after surgery (to allow for tracer transport), birds were overdosed with Equithesin and perfused intracardially with PBS (20 ml), followed by ice-cold 4\% PFA (40 ml). Dissected brains were postfixed overnight in $4 \%$ PFA, followed by PBS until sectioning. Brains were sectioned $(40 \mu \mathrm{m})$ in the sagittal or coronal plane using a vibratome.

Imaging of extrinsic labeling. In preparation for imaging, sections were mounted on microscope slides and coverslipped with saline. Fluorescent imaging was done using the same microscope/computer system described above. Using either rhodamine (DiI) or fluorescein (DiO) optics, digital image stacks $(20 \times$ objective) of retrograde labeling in regions that provide major sources of afferent input to HVC (nucleus interfacialis [NIf]; medial magnocellular nucleus of the anterior nidopallium [MMAN]; nucleus uvaeformis [Uva]) were captured at $5 \mu \mathrm{m}$ increments and then collapsed into single images. We also documented the presence of retrogradely labeled cells in two smaller sources of HVC afferent input: the dorsal region of RA and nucleus Avalanche (Av). Efferent labeling of HVC axons was captured by creating composite images of the caudal telencephalon $(5 \times$ objective). After imaging, mounted sections were stained with thionin and imaged again under brightfield illumination to visualize the Nissl-defined borders of NIf, MMAN, and Uva.

Placement of tracer injection site in HVC. Tracer injection sites were imaged to confirm their location within the Nissl-defined borders of HVC. Centered on the injection sites, radial diffusion of tracer ranged from 0.25 to $0.45 \mathrm{~mm}$ (average, $0.39 \mathrm{~mm}$ ). In birds with a large distance between injection sites $(\geq 0.8 \mathrm{~mm}, N=2)$, radial spread of medial and lateral tracers did not overlap (average gap of $0.24 \mathrm{~mm}$ ). Birds with closer injection sites ( $\leq 0.6 \mathrm{~mm}, N=5)$ showed increasing overlap as the distance between injection sites decreased.

Quantification of retrograde labeling. Digital images from NIf, MMAN, Uva, RA, and Av were blended to reveal the presence of double-labeled (yellow) cells. We used Neurolucida software to quantify the incidence of single- or double-labeled cells in NIf, MMAN, and Uva. MMAN was lost in one bird during tissue processing. Thus, the final sample sizes for each region were as follows: NIf, $N=7$; MMAN, $N=6$; Uva, $N=7$. Because of the low overall number of labeled cells in dorsal RA and in Av, the pattern of double- and single-labeling in these regions was assessed visually.

\section{Statistical analyses}

HVC ablation extent was analyzed by one-way ANOVA to determine whether the volume of HVC ablated varied between HVC subregion ablation groups. A $t$ test was used to determine that HVC ablations were bilaterally symmetrical. LMAN volume remaining within the HVC subregion ablation groups was assessed by one-way ANOVA using the percentage remaining LMAN values for each group. Composite KL distance values were analyzed by one-way ANOVA followed by Bonferroni post hoc tests comparing each group to CTL. All syllable production and transition analyses were analyzed by two-way ANOVAs followed by Bonferroni post hoc tests.

\section{Results \\ HVC subregion ablation extent and location}

HVC subregions (MED, CENT, LAT) were targeted for bilateral ablation in each bird by customizing the number of electrode penetrations and current durations to the size and shape of each bird's HVC. HVC is bilaterally symmetrical (Fig. 1B), and the number of electrode penetrations and current durations were therefore mirrored across hemispheres in each bird. The extent and location of HVC subregion ablations were quantified by retrograde labeling of HVCX neurons with DiI to unambiguously delineate the borders of HVC. For example, Figure $3 A$ shows a series of sagittal tissue sections from a bird that received bilateral MED HVC ablation: the images show DiI-labeled HVCX neurons in LAT and CENT HVC, whereas an autofluorescent scar is present in the location of MED HVC. In CENT and LAT HVC ablation groups, scar tissue is present only in CENT HVC (Fig. $3 B$ ) or in LAT HVC (Fig. 3C).

Three-dimensional reconstruction of each HVC in each bird revealed that unilateral ablation volume did not vary as a function of the HVC subregion that was targeted $\left(F_{(2,29)}=1.22, p=0.31\right)$. Moreover, the volumes of HVC subregion ablations did not vary significantly between hemispheres $\left(t_{(30)}=0.55, p=0.59\right)$. The mean \pm SEM unilateral volumes of HVC subregion ablations were $0.039 \pm 0.012 \mathrm{~mm}^{3}$ for the medial group, $0.026 \pm 0.014$ $\mathrm{mm}^{3}$ for the central group, and $0.054 \pm 0.013 \mathrm{~mm}^{3}$ for the lateral group. Thus, the location, and not the amount, of HVC ablation was the independent variable in this experiment.

\section{Extent of LMAN ablation}

Bilateral LMAN ablation was necessary to isolate HVC premotor drive on song. LMAN ablations were verified in each bird by reconstructing any remaining LMAN visible in Nissl-stained serial sections. If no LMAN was visible, ablation was estimated at $100 \%$. For each hemisphere, remaining LMAN volume was divided by an average unilateral LMAN volume for adult birds $\left(0.11 \mathrm{~mm}^{3}\right)$ to estimate the percentage of remaining LMAN. For the HVC ablation groups, percentage LMAN remaining did not vary significantly $\left(F_{(2,13)}=0.173, p=0.84\right)$. The mean \pm SEM percentage of remaining LMAN was $1.8 \pm 1.8 \%$ for the medial group, $4.0 \pm 2.5 \%$ for the central group, and $2.4 \pm 2.4 \%$ for the lateral group. Birds with $>25 \%$ LMAN remaining, either purposefully or as the result of an ablation miss, comprised the LMANR group of $N=6$ birds that also underwent HVC subregion ablation surgery. These birds had a mean \pm SEM of $85.4 \pm$ $11.8 \%$ LMAN remaining. Thus, only LMANR birds retained a second source of premotor drive on vocal output.

\section{In the absence of LMAN, ablation of HVC subregions does not cause deficits in the acoustic structure of song syllables}

Figure 4 compares syllable duration versus syllable pitch scatterplots from PRE1 and POST3 singing in representative birds from each experimental group. Within each scatterplot, each colored cluster represents multiple instances $(>100)$ of an individual syllable recorded over the course of PRE1 (pale colors) or POST3 (saturated colors). Figure $4 A$ shows an example of the consequences of bilateral HVC subregion ablation when LMAN remains intact. Syllable clustering is lost at POST (black data points) as song reflects the unstructured acoustic character of LMAN-driven singing, which is unmasked by damage to HVC (Thompson and Johnson, 2007; Thompson et al., 2007; Aronov et al., 2008). However, if LMAN is ablated, syllable clustering is preserved following bilateral CTL, MED, or CENT HVC ablations (Fig. $4 B-D$ ). Figure $4 E$ shows an example of the vocal effects of bilateral ablation of LAT HVC. Syllables produced by LAT HVC birds at POST also retain the acoustic features of their PRE counterparts, but some syllables are omitted at POST (syllables C and $\mathrm{D}$ in this example).

Differences between scatterplots of PRE and POST singing were quantified by KL distance, a metric derived from probability and information theory that quantifies the difference in bits between two probability density distributions (in our case, two scat- 


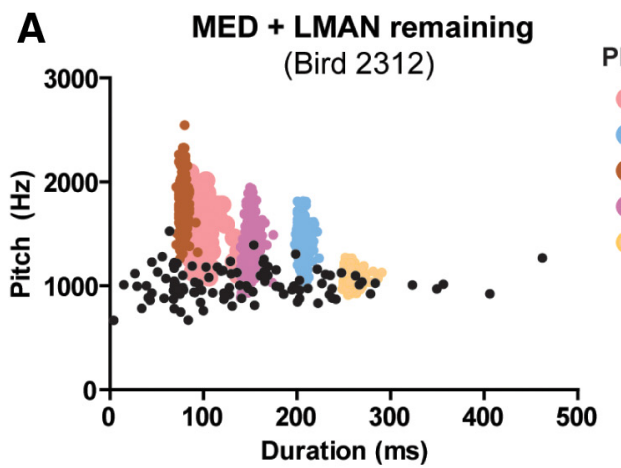

B

CTL

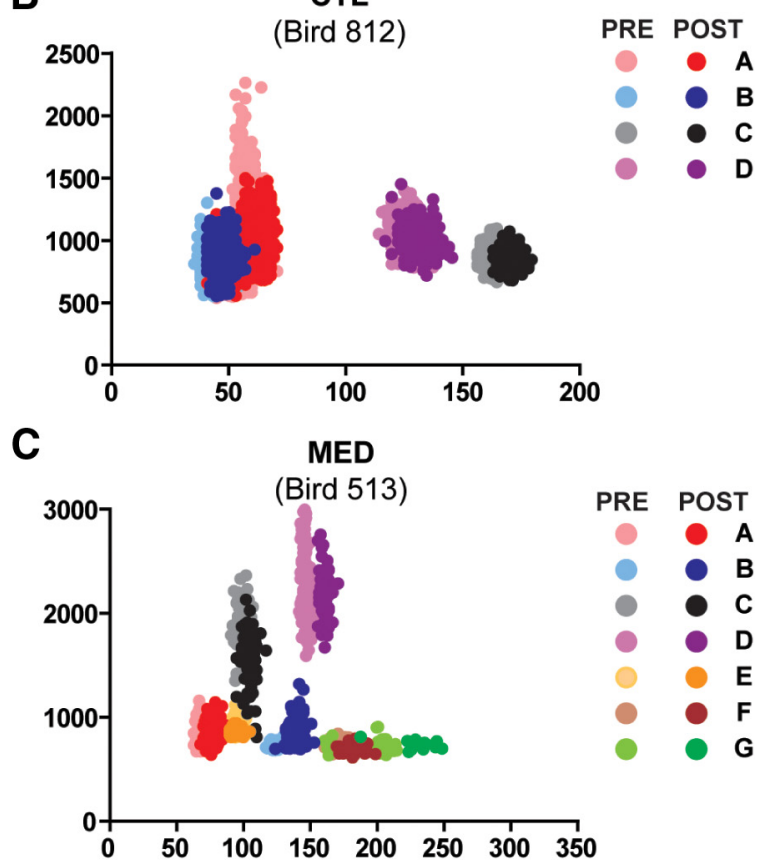

D

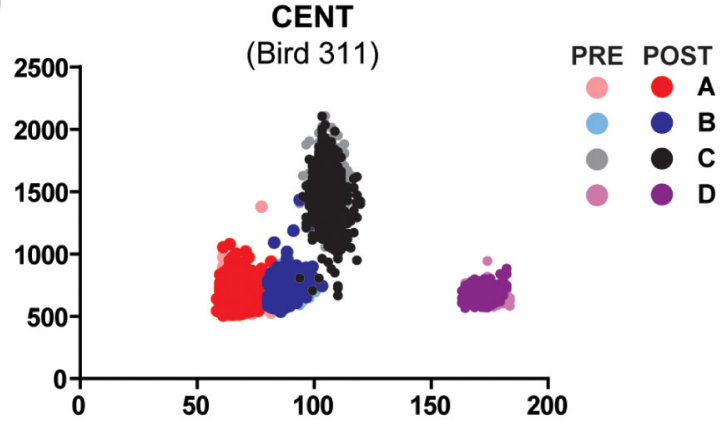

E

LAT

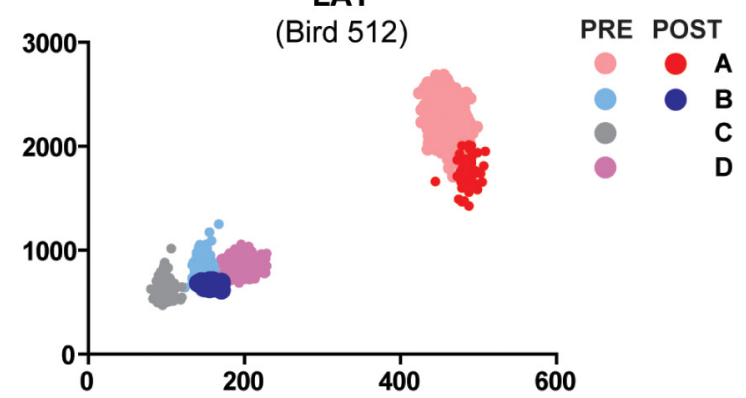

Figure 4. In the absence of LMAN, bilateral ablation of HVC subregions does not produce a general disruption of syllable acoustic structure. $A$, In the presence of $L M A N$, bilateral damage to

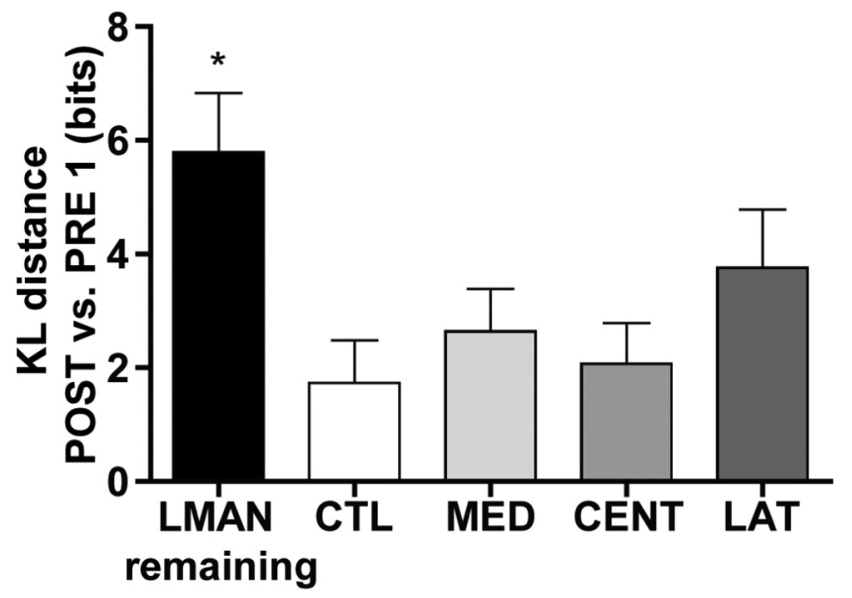

Figure 5. Quantification of changes in syllable acoustic features after HVC subregion ablation by group. Values are Kullback-Leibler (KL) distances of all POST singing from PRE1, calculated using a composite measure of syllable acoustic features (duration, pitch, FM, entropy, and pitch goodness). Data are the mean \pm SEM of these values within groups. Only birds with LMAN remaining were significantly different from CTL birds $\left({ }^{*} p<0.05\right)$. A nonsignificant trend is observed when LAT birds are compared with CTL birds $(p=0.15)$.

terplots) (for additional details, see Thompson et al., 2007; Wu et al., 2008; Materials and Methods). Using scatterplots generated from four different acoustic feature pairs from each bird (syllable duration vs syllable pitch, syllable duration vs syllable FM, syllable duration vs syllable entropy, syllable duration vs syllable pitch goodness), differences in the structure of PRE1 singing and the structure of POST1-3 singing were quantified by KL distance. In each bird, all four acoustic feature pair comparisons produced similar results that were consistent across the 3 POST days. We therefore averaged across feature pairs and POST days to generate a single composite KL distance value for the POST singing of each bird. Figure 5 shows that, although this composite measure of KL distance varied as a function of experimental group $\left(F_{(4,22)}=\right.$ 3.77, $p<0.05$ ), only LMANR birds (birds in which LMAN remained intact) were significantly different from CTL birds $(p<$ 0.05). Together, the data in Figures 4 and 5 indicate that ablation of HVC subregions does not produce a statistically significant disruption to the acoustic structure of postoperative song syllables, as long as this damage occurs in the absence of LMAN.

Damage immediately surrounding HVC does not affect the sequence or structure of song

Some of the birds in the HVC subregion ablation groups received damage that extended past the lateral, ventral, or medial borders of HVC. To control for the effects of damage to neural tissue immediately surrounding HVC, CTL birds underwent surgical procedures that mimicked subregion ablations, but ablation electrodes were placed outside of HVC. Figure 6 shows example spectrograms from two birds that received damage to neural tissue immediately medial (A) or ventral (B) to HVC. Neither bird

medial HVC results in a complete loss of syllable acoustic structure (compare with spectrograms in Fig. 2). $\boldsymbol{B}$, In the absence of LMAN, bilateral damage that flanks but does not include HVC has a minimal effect on syllable acoustic structure (compare with spectrograms in Fig. 6). $\boldsymbol{C}$, In the absence of LMAN, bilateral damage to medial HVC has a minimal effect on syllable acoustic structure (compare with spectrograms in Fig. 7). D, In the absence of LMAN, bilateral damage to central HVC has a minimal effect on syllable acoustic structure. $\boldsymbol{E}$, In the absence of LMAN, bilateral damage to lateral HVC results in omission of some syllables $(\boldsymbol{C}, \boldsymbol{D})$ but has minimal effect on the acoustic structure of remaining syllables ( $A, B$, compare with spectrograms in Fig. 7). 
showed a change in the acoustic structure of song syllables at POST. Importantly, syllable sequence was also preserved after surgery, as shown in the syllable transition diagrams located to the right of the spectrograms (grayscaling is used to illustrate the relative probability of each syllable transition within the motif).

The absence of vocal disruption at POST was common to all CTL birds regardless of ablation location around HVC. This included birds where damage encompassed paraHVC, a region that contains neurons that project to Area $\mathrm{X}$ but does not contain any neurons that project to RA (Johnson and Bottjer, 1995; Foster and Bottjer, 1998). The average bilateral volume of ablated tissue in this group was $0.15 \pm 0.06 \mathrm{~mm}^{3}$. These findings in CTL birds indicate that the effects seen in MED, CENT, and LAT HVC groups are not due to any effect of surgery alone, but to the specific location of damage within HVC.

\section{Location of HVC damage differentially affects the sequence and structure of song}

After ablation surgery, birds in the LAT HVC group displayed a negative deficit (omission of one or more syllables), whereas birds in the MED HVC group displayed a positive deficit (an increase in atypical syllable sequences). Figure 7 shows examples of these song deficits in representative birds from LAT and MED HVC groups. Figure $7 A$ shows a bird with a canonical motif of A-B-C-D at PRE. After LAT HVC surgery, the motif is truncated ( $\mathrm{C}$ and $\mathrm{D}$ are omitted). This can be observed in the POST spectrogram and the syllable transition diagrams to the right. At PRE, relative probabilities are evenly distributed across all syllable transition types due to the serial production of motif syllables. At POST, all transitions are now between $\mathrm{A}$ and $\mathrm{B}$ ( $\mathrm{C}$ and $\mathrm{D}$ were omitted after surgery), resulting in a single, black arrow between syllables A and B. Figure $7 B$ depicts another LAT HVC bird that omitted three syllables at the end of its motif after surgery. As with the bird depicted in Figure $7 A$, this bird produced only one syllable transition type after surgery (A-B), as the bird omitted syllables $\mathrm{C}-\mathrm{E}$ from its repertoire at POST. The omission of motif syllables at POST was characteristic of birds with LAT HVC ablations and was wholesale in 3 of 5 birds in this group.

Figure $7 C$ shows the singing of a bird that received MED HVC surgery. This bird continues to produce its full PRE rep-
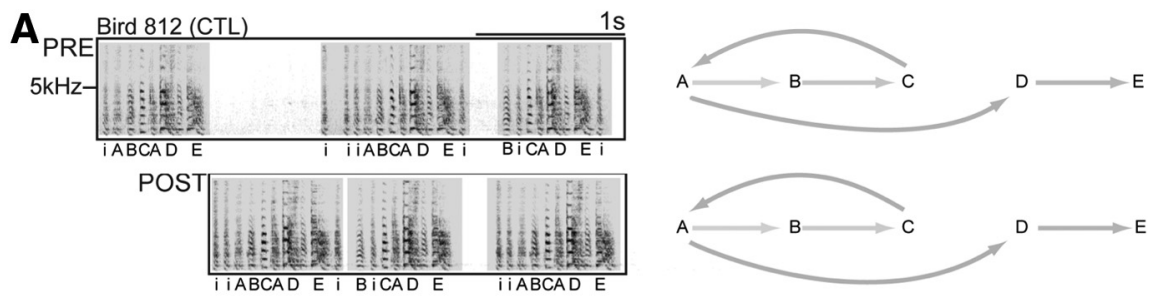

B

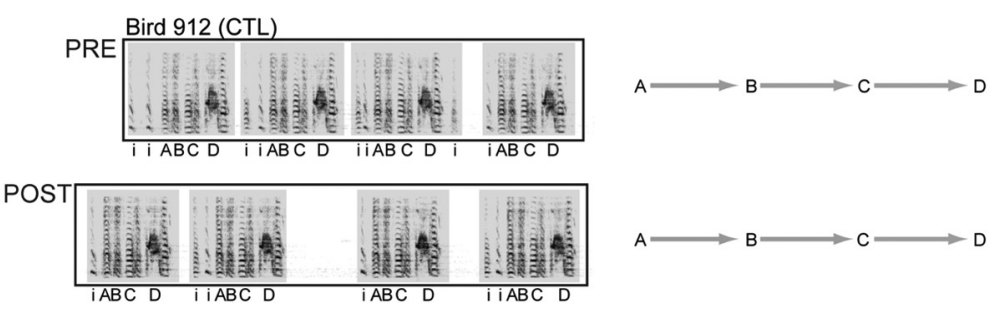

Figure 6. Bilateral damage that flanks HVC borders does not affect the motor encoding of song. $A, B$, Example spectrograms show that birds sing complete motifs at POST when bilateral damage does not include HVC. Bird 812 had damage located medial and ventral to HVC. Bird 912 had damage located ventral to the central portion of HVC. Syllable transition diagrams accompanying each spectrogram show the changes in syllable transition types associated with this deficit. Grayscale represents the relative probabilities of transitions between pairs of motif syllables.

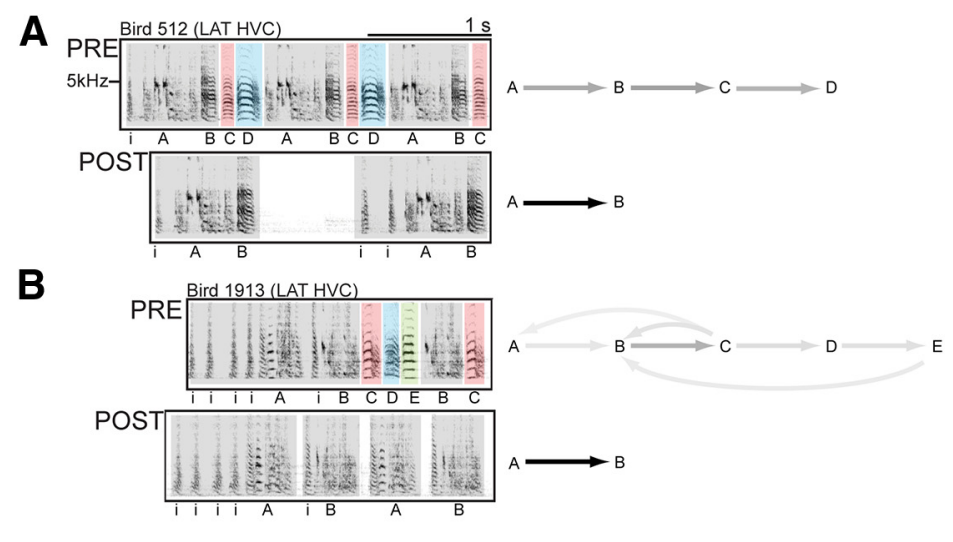

C

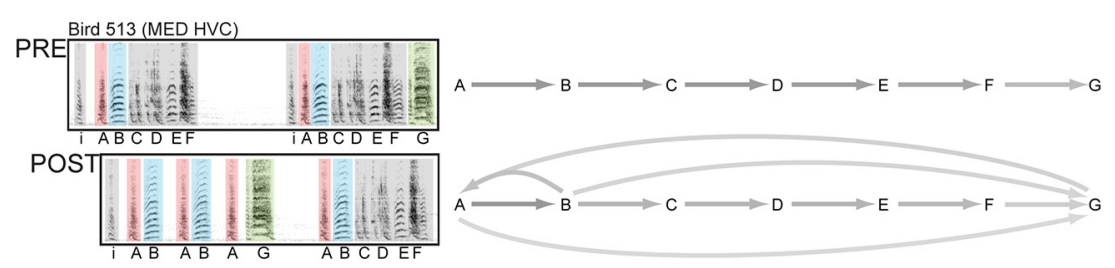

D

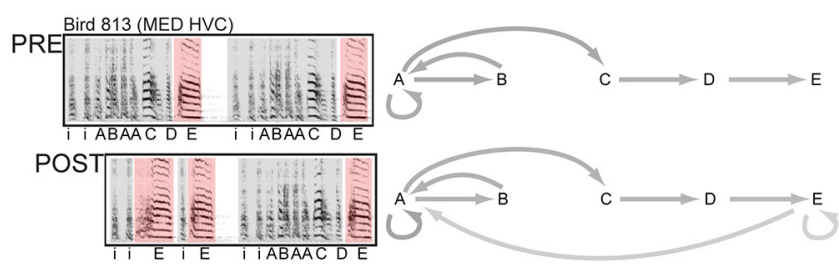

Figure 7. LAT and MED HVC ablations affect singing differently. $\boldsymbol{A}, \boldsymbol{B}$, Example spectrograms show that birds omit syllables at POST when LAT HVC is bilaterally damaged, resulting in incomplete motifs. Syllable transition diagrams accompanying each spectrogram show the changes in syllable transition types associated with this deficit. Grayscale represents the relative probabilities of transitions between pairs of motif syllables. At PRE, different proportions of the transitions throughout a day of singing correspond to transitions between pairs of motif syllables. At POST, all transitions now occur between syllables $A$ and B because only those two syllables were sung by these two birds. The omission of motif syllables at POST was characteristic of birds with LAT HVC ablations and was wholesale in 3 of 5 birds in this group. C, D, Example spectrograms and syllable transition diagrams show that birds with bilateral MED HVC damage sing all of their motif syllables at POST, but they intermittently produce their motif syllables out of the canonical order observed at PRE. New and atypical transition types are present at POST (e.g., B-A and A-G at POST in C). Atypical syllable transitions were characteristic of birds with MED HVC ablations, although this deficit was never wholesale: all birds in this group retained the ability to intermittently produce their canonical PRE motif. 

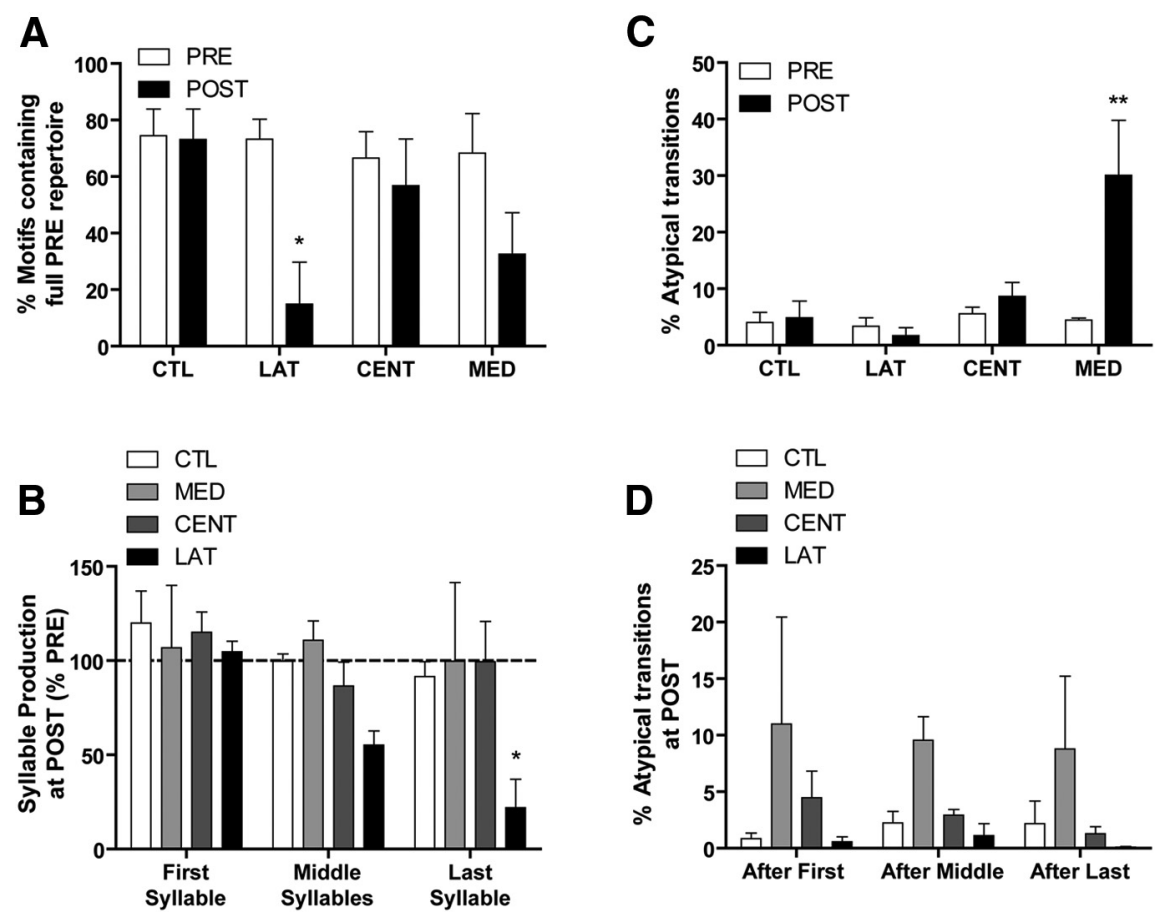

Figure 8. Quantification of syllable omission and atypical syllable transitions, as well as the motif position (first, middle, or last syllables) of the two types of vocal disruption. Data are mean \pm SEM. A, Quantification of motif completion rates reveals which experimental groups continued to sing their full PRE repertoire after surgery. At PRE, all groups completed their motifs (i.e., the motif included the full PRE repertoire of syllables) between two-thirds and three-fourths of the time. Only the LAT HVC group showed a significant decrease in complete motifs at POST ( ${ }^{*} p<0.05$ vs PRE), indicative of the omission of motif syllables. $\boldsymbol{B}$, During POST singing, only LAT HVC birds showed a specific pattern in syllable omission. Syllables were selectively omitted at the end of the motif. C, Quantification of atypical syllable transitions reveals which experimental groups diverged from their canonical ordering of syllables after surgery. Atypical transitions resulting from syllable omissions were not quantified here. Only the MED HVC group showed a significant increase in atypical transitions at POST $\left(^{* *} p<0.01\right)$. D, During post singing, no experimental group showed a pattern in atypical transitions, which even in the MED HVC group were just as likely to follow the first, middle, or last syllables of the motif.

ertoire of syllables at POST; however, the canonical sequence of syllables is disrupted in some motifs. Syllables A and B are repeated many times, and the final syllable, G, is sung immediately after a rendition of syllable $\mathrm{A}$, and before a canonical motif. This change in POST singing is depicted in the syllable transition diagrams to the right. Atypical transitions between syllables appear at POST (e.g., B-A and A-G), which proportionally reduces the grayscaling of some typical syllable transitions (e.g., B-C or C-D). A less dramatic change in sequence is observed in Figure $7 D$, where a bird that also received MED HVC surgery misplaces its final syllable, E. The bird sings syllable E multiple times, divorced from the syllable's canonical position in the motif, before going on to complete a canonical motif. Again, this deficit is depicted in the syllable transition diagram with new arrows at POST from E-A and E-E. Atypical syllable transitions were characteristic of birds with MED HVC ablations, although this deficit was never wholesale: all birds in this group retained the ability to intermittently produce their canonical PRE motif.

When present, vocal deficits following CENT HVC ablation resembled those that followed LAT HVC ablation. That is, no bird that received bilateral ablation of CENT HVC showed the type of positive deficit (increase in atypical syllable sequences) observed following MED HVC ablation (Fig. 7C,D). Indeed, 3 of 6 CENT HVC birds showed no significant change in their POST singing, a replication of the work of Poole et al. (2012) who observed no significant change in the sequence or structure of song following knife-cut transections across the rostral-caudal axis of
CENT HVC. However, our electrolytic ablations of CENT HVC, which produced a greater region of damage than the knifecut transections used by Poole et al. (2012), caused syllable omission in 3 of 6 CENT HVC birds. The negative disruption in this subset of CENT HVC birds was therefore similar to the effects of LAT HVC ablation (Fig. $7 A, B$ ).

\section{Lateral HVC ablation induces syllable omission at the end of the motif}

To compare and quantify the negative (LAT) and positive (MED) deficits produced by ablation of different portions of HVC, we made PRE versus POST comparisons of the percentage of motifs containing the full PRE repertoire of syllables. Although it is normal for adult male zebra finches to truncate a portion of their motifs (observed in this study to be $\sim 30 \%$ at PRE), LAT HVC ablation appeared to dramatically increase the number of motifs with omitted syllables. Figure $8 \mathrm{~A}$ shows the result of this comparison, which produced a significant main effect of experimental group $\left(F_{(1,34)}=8.40, p<\right.$ $0.01)$. Moreover, only the LAT HVC group showed a significant decrease in the percentage of motifs containing the full PRE repertoire at POST $(p<0.05)$. Although MED HVC birds sang motifs that included only a portion of their PRE repertoire, often arranged in atypical sequences, this did not occur with sufficient frequency to significantly decrease the percentage of motifs containing the full PRE repertoire at POST $(p=0.1)$

We then asked whether omission of syllables at POST occurred at a particular position within the motif. Figure $8 B$ depicts the results of an analysis where syllable production was quantified as a function of syllable position within the motif (first, middle, last). Compared with PRE singing, omission of syllables at POST varied as a function of experimental group $\left(F_{(3,50)}=3.66, p<\right.$ $0.05)$ as well as syllable position within the motif $\left(F_{(2,50)}=3.26\right.$, $p<0.05)$. Compared with CTL birds, a trend of increasing syllable omission by LAT HVC birds is apparent across middle and last motif syllables at POST. However, only omission of the last motif syllable by LAT HVC birds reached statistical significance ( $p<0.05, p=0.17$ for middle syllables). In contrast, production of first, middle, and last syllables by MED HVC birds did not differ between PRE and POST singing. Although MED HVC birds sang some motifs at POST that included only a portion of their PRE repertoire (Fig. $8 A$ ), the omitted syllables did not vary as a function of position within the motif, and syllable omission was balanced by an increase in the production of other motif syllables. Thus, the average production of first, middle, and last syllables by MED HVC birds at POST remained close to PRE levels (Fig. $8 B$ ). These data highlight the behavioral difference between MED and LAT HVC birds and indicate that the negative vocal deficit observed following LAT HVC ablation involves the selective omission of syllables toward the end of the motif. 
Medial HVC ablation induces atypical syllable transitions at all motif positions

In the context of a canonical motif, atypical syllable transitions could occur when syllables are omitted (e.g., A-B-C-D becomes $\mathrm{A}-\mathrm{B}-\mathrm{D}$ if $\mathrm{C}$ is omitted) or when there is a change to the canonical ordering of syllables (e.g., A-B-C-D becomes D-AA-C-B). Whereas atypical transitions associated with syllable omission could be viewed as adaptive (the sequence proceeds normally in the absence of one of the elements), a change in canonical ordering would be evidence of a true disruption of the mechanisms of syllable sequencing. To selectively analyze a disruption of canonical ordering, we measured atypical transitions using the repertoire of syllables that each bird produced at POST. This effectively removed atypical transitions due to syllable omission that would otherwise be detected in the POST singing of HVC LAT birds. Moreover, only bouts of singing that contained at least one instance of each bird's full POST repertoire were analyzed.

Figure $8 C$ shows PRE versus POST comparisons of the percentage of atypical syllable transitions for each experimental group. Syllable transitions classified as atypical were found to vary as a function of the interaction between experimental group and surgery (PRE vs POST; $F_{(3,34)}=$ $5.62, p<0.01)$. We then found that a significant postoperative increase in atypical transitions, specifically those that cannot be accounted for by syllable omission, occurred only in the MED HVC group $(p<0.01)$.

As with syllable omissions in HVC LAT birds, we then asked whether the atypical transitions of MED HVC birds occurred consistently at a particular position within the motif. Figure $8 D$ depicts the results of an analysis where atypical transitions at POST are quantified as a function of whether the atypical transition occurred after the first, middle, or last syllables in the motif. As expected, the percentage of syllable transitions classified as atypical was greater in the MED HVC group $\left(F_{(3,50)}=4.13, p<0.05\right)$, but there were no differences as a function of whether the atypical transition occurred after the first, middle, or last syllables of the motif $(F<1)$. These data further highlight the behavioral difference between MED and LAT HVC birds and indicate that the positive vocal deficit observed following MED HVC ablation involves an increase in atypical transitions that were just as likely to occur after the first, middle, or last syllables of the motif.
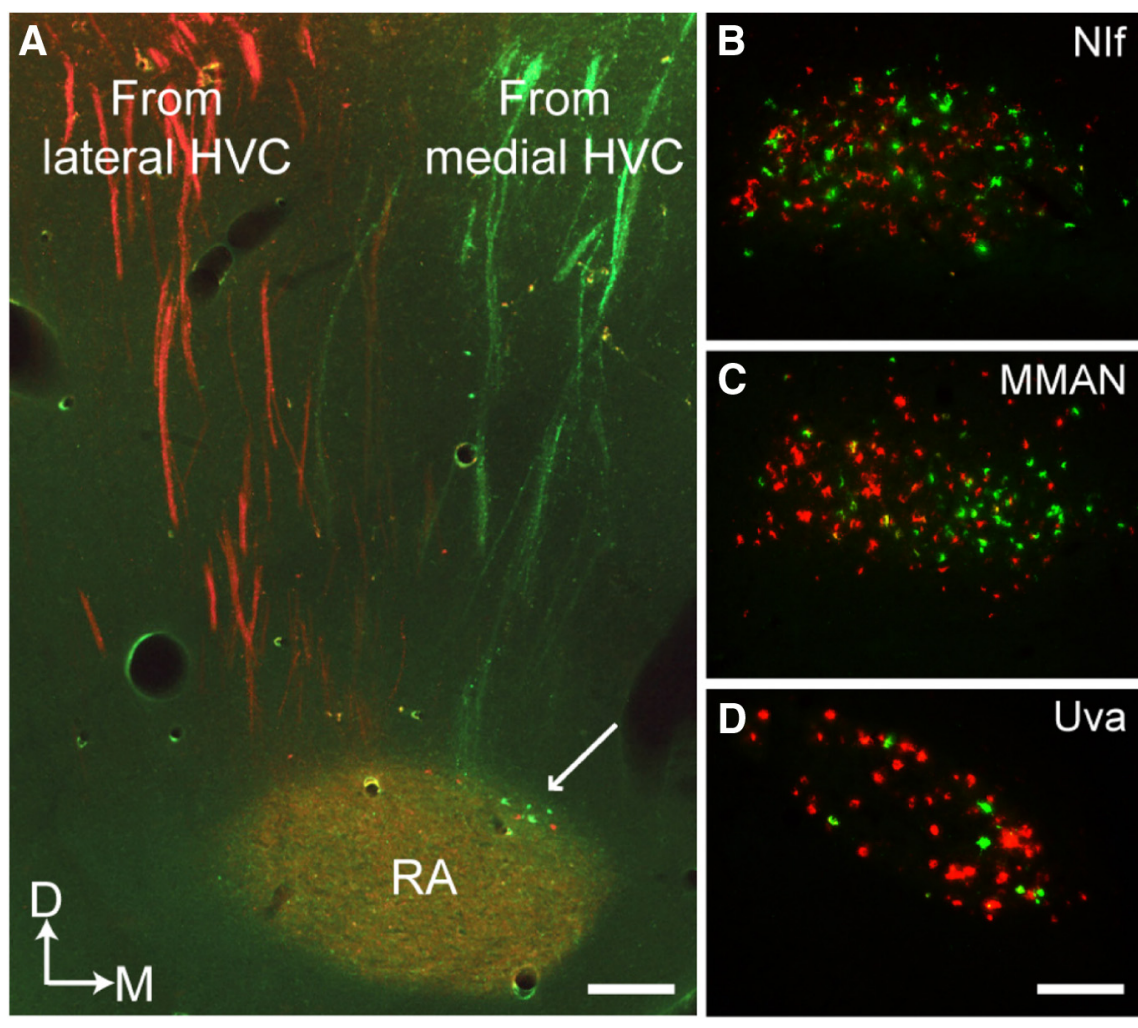

E

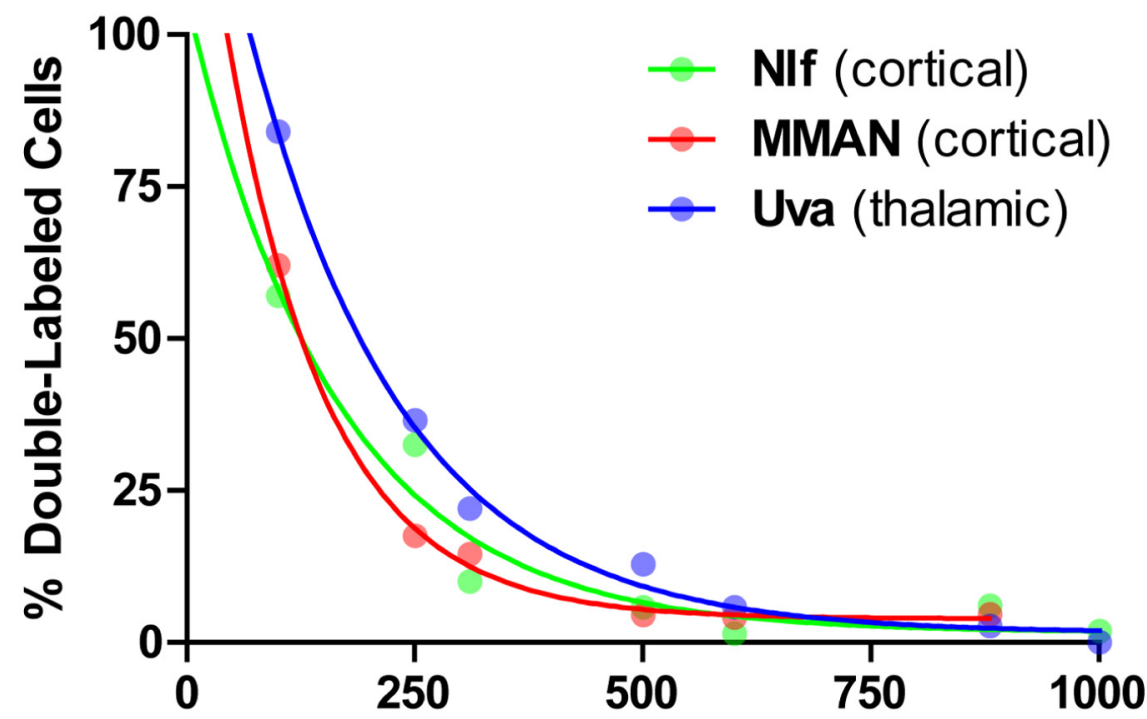

\section{Distance Between HVC Injection Sites ( $\mu \mathrm{m})$}

Figure 9. Parallel organization of HVC extrinsic connectivity. Tracer injections in medial (Di0, green) and lateral (Dil, red) HVC show that the extrinsic connectivity of HVC supports a partitioning of vocal function across medial and lateral portions of HVC. Images show that medial and lateral HVC send axons to RA in distinct pathways $(\boldsymbol{A})$ and receive distinct sources of afferent input from NIf, MMAN, and UVA $(\boldsymbol{B}-\boldsymbol{D})$. This pattern of anterograde and retrograde labeling was observed in all birds $(N=4)$ that received dye injections into medial and lateral HVC. $\boldsymbol{A}$, Arrow indicates the small population of neurons in dorsal RA that are reciprocally connected to HVC (Roberts et al., 2008). These cells were also found to project medial HVC (green) or lateral HVC (red), but not both. Scale bars: $A, 165 \mu \mathrm{m} ; \boldsymbol{D}, 120 \mu \mathrm{m}$. E, Demonstration of experimental control over tracer labeling. Double-labeling of neurons in HVC afferent nuclei (NIf, MMAN, Uva) is an exponential function of the medial-lateral distance between $\leq 40 \mathrm{nl}$ Dil and DiO injections in HVC. Data are single hemisphere values from $N=7$ birds. The exponential function indicates that axon terminals from NIf, MMAN, and Uva neurons are narrowly targeted to either medial or lateral HVC. 


\section{HVC extrinsic connectivity is organized into medial and lateral parallel pathways}

The results from our HVC subregion ablation experiment suggest that medial and lateral HVC play independent, potentially parallel roles in encoding syllable sequence and syllable structure, respectively. This view is affirmed by the observation that disconnecting medial from lateral HVC via knife-cut transection is without effect on the sequence or structure of song (Poole et al., 2012). To function independently, one would presume that medial and lateral HVC should receive independent sources of afferent input. However, prior anatomical studies using the injection of a single tract-tracing dye into HVC suggest that afferent input to HVC is nontopographic and convergent (Bottjer et al., 1989; Fortune and Margoliash, 1995; Foster and Bottjer, 1998). Motivated by our behavioral findings, we reexamined the afferent connectivity of HVC using two different tract-tracing dyes: one placed in medial HVC and one placed in lateral HVC. By using dyes that are transported in both retrograde and anterograde directions (DiI and DiO), efferent pathways emanating from medial and lateral HVC were also examined in the same birds.

Figure $9 A-D$ summarizes afferent and efferent labeling from a bird where DiI (lateral HVC) and DiO (medial HVC) injections were separated by $880 \mu \mathrm{m}$. Figure $9 A$ shows a coronal image of HVC efferent axons and their target (RA), where only one dye (red or green) was present in any axon bundle, indicating that the labeled efferent projections originate from either medial or lateral HVC. However, a convergence of medial and lateral efferent projections is observed within RA, where red and green axon terminals mix. This pattern of labeling suggests that parallel streams of neural activity arising from medial and lateral HVC converge and are integrated at the level of vocal-motor cortex, RA. Figure 9A (arrow) also shows the small population of dorsal RA neurons that project back to HVC (Roberts et al., 2008). These cells are labeled with only one dye (red or green), indicating that RA-HVC reciprocal connectivity is also organized in parallel.

Figure $9 B-D$ shows the pattern of retrograde labeling in three major sources of HVC afferent input: the cortical regions NIf and MMAN, and the thalamic region Uva. The vast majority of cells in each region are labeled with only one dye or the other, indicating that, with few exceptions, afferent projections target either lateral or medial HVC, not both. In this bird, the incidence of double-labeled cells in NIf, MMAN, and Uva was 6\%, 4.6\%, and $2.7 \%$, respectively. We also examined retrograde labeling in Av, a small population of cortical neurons that also provides afferent input to HVC, and it also showed the same pattern of largely single-labeled cells as NIf, MMAN, and Uva (data not shown).

We then varied the distance between $\mathrm{DiI}$ and $\mathrm{DiO}$ injections in additional birds to demonstrate experimental control over single versus double-labeling of cells in NIf, MMAN, and Uva. The results of this analysis are shown in Figure 9E, where the incidence of double-labeling in each afferent region decreases exponentially as the distance between injection sites within HVC increases from 0.1 to $1.0 \mathrm{~mm}$. The exponential function suggests that the terminal arborizations of NIf, MMAN, and Uva neurons are narrowly targeted to specific regions of HVC, adding further support to the conclusion that the extrinsic connectivity of HVC is organized into anatomically distinct medial and lateral streams, a circuit organization consistent with the observed independent vocal functions of medial and lateral HVC.

The HVC dye injections also labeled the terminals of HVCX neurons. The pattern of terminal labeling in Area X was similar to that observed in the HVC-RA pathway, meaning that terminal labeling filled the volume of Area X regardless of whether dye was injected into medial or lateral HVC (data not shown). This lack of topography in terminal arborizations of HVCX neurons confirms the work of Luo et al. (2001) who injected two different retrograde tracers into different regions of Area X and observed double-labeling of HVCX neurons in all instances.

\section{Uva neurons preferentially target lateral HVC}

The distributions of single-labeled cells were nontopographic in NIf and Uva, whereas MMAN showed a subtle gradient of mediolateral topography. Given this generally nontopographic pattern of retrograde labeling, it is understandable why experiments using only a single-dye injection in HVC would conclude that HVC receives a nontopographic and convergent pattern of afferent input (Fortune and Margoliash, 1995; Foster and Bottjer, 1998). Interestingly, although injections of dyes into medial and lateral HVC labeled similarly sized populations of cells in NIf and MMAN, we found that lateral HVC dye injections consistently labeled more Uva cells than medial HVC dye injections. This can be observed in the image of Uva shown in Figure 9D, where there are many more cells labeled by the lateral HVC injection (red) than the medial HVC injection (green). To quantify this apparent difference, we calculated the average ratio of Uva neurons that projected either to lateral or to medial HVC.

We restricted this analysis to a subset of birds where the vast majority of cells were single-labeled (i.e., the distance between the two injection sites was $\geq 600 \mu \mathrm{m}, N=3$ birds; Fig. $9 E$ ). Compared with the average ratio of NIf and MMAN neurons that projected either to lateral or to medial HVC, we found that Uva neurons were 2.3 times more likely to project to lateral than medial HVC. This result suggests that Uva contains a much larger proportion of neurons projecting to lateral HVC than NIf or MMAN, a possible indication that Uva exerts a greater influence on lateral HVC activity and further evidence of a functional difference between medial and lateral HVC.

\section{Discussion}

HVC premotor activity encodes the learned song pattern (Long and Fee, 2008; Goldin et al., 2013), but little is known about how premotor activity at any given HVC location contributes to the song pattern as a whole. Arguably, it has been implicitly assumed that HVC premotor encoding is globally distributed, similar to the sensory memory for autogenous song that is also encoded within HVC (Sutter and Margoliash, 1994; Graber et al., 2013). Consequently, the anatomical organization of the temporally organized neural activity recorded from HVC in singing birds has never been documented (Yu and Margoliash, 1996; Hahnloser et al., 2002; Kozhevnikov and Fee, 2007; Long et al., 2010; Fujimoto et al., 2011; Amador et al., 2013; Hamaguchi et al., 2014).

Here we tested the hypothesis that medial and lateral HVC encode different dimensions of the song pattern. This was accomplished by first ablating LMAN bilaterally (so that singing was under premotor control of HVC alone) (Thompson et al., $2007,2011)$ and then bilaterally ablating portions of medial, central, or lateral HVC and observing the effect on song. We found that ablation within medial HVC caused a positive disruption of song (increase in atypical syllable sequences), whereas ablation within lateral HVC caused a negative disruption of song (omission of one or more syllables at the end of the motif). The contrasting positive/negative vocal disruption that followed ablation within medial or lateral HVC argues against a globally distributed encoding of song within HVC. Interestingly, ablation within cen- 
tral HVC either had no effect (compare Poole et al., 2012) or produced the omission of syllables from the song pattern (3 of 6 birds), similar to lateral HVC ablation. Consistent with prior observations that the medial-lateral connectivity of HVC is limited (Stauffer et al., 2012), all ablation-induced deficits were isolated and specific: in the absence of LMAN, general degradation across the entire song pattern was never observed following ablation within any HVC subregion.

Figure 10 summarizes all HVC ablation loci that produced atypical sequencing (green) or syllable omission (red), or had no effect (dashed lines). This mapping suggests a functional topography within HVC, with the medial third encoding and/or accessing syllable sequence and the lateral two-thirds encoding and/or accessing syllable structure. If medial and lateral regions of HVC function independently, it would follow that HVC extrinsic connectivity should be organized in parallel. Although prior work using injections of a single tracer indicated a parallel organization for the HVC-RA axon pathway (Foster and Bottjer, 1998), we used injections of dyes into medial and lateral HVC to further demonstrate that all cortical and thalamic afferent inputs to HVC are also organized in parallel. We also noted an intriguing asymmetry in afferent input to HVC: the thalamic region Uva preferentially targets lateral HVC, potentially indicative of a greater role for this region in modulating the structure of song syllables.

As with any ablation study, our findings reflect the necessity, but not the sufficiency, of the ablated subregions for syllable sequence and/or production. Moreover, because each ablated subregion contained portions of all three HVC neuron populations (HVCX, HVCRA, and HVCINT), we cannot address the hypothesized role of HVCX neurons in syllable sequence (Fujimoto et al., 2011; Hamaguchi et al., 2014) or the role of HVCRA neurons in syllable structure (Hahnloser et al., 2002; Long et al., 2010; Amador et al., 2013). Ablated portions of HVC could serve a role in encoding unaffected syllables or sequence, and intact portions of HVC could still serve a role in the encoding affected syllables or sequence. Interestingly, following lateral HVC ablations, the omission of spectrally complex syllables was never observed. Instances of spectrally complex syllables are seen in Figure 7A (syllable A) and Figure $7 B$ (syllables A and B). One possibility is that these syllable types may be encoded more frequently or more globally than others within HVC. Thus, although our findings do not address a possible topography of individual syllables within HVC, they may provide a useful guide for such work. In this regard, it should be noted that individual elements of the song pattern are distributed across left and right HVC (Ashmore et al., 2008; Long and Fee, 2008; Wang et al., 2008).

\section{Computational models of HVC encoding of song}

As noted above, prior studies of HVC neural activity in singing birds did not examine the anatomical location of the recorded neurons. Therefore, computational models of HVC that are informed by these recordings do not include an anatomical organization. All are associative or probabilistic chain models, where the sequence and structure of song are encoded in a unitary fashion and where successful completion of one syllable provides the stimulation that drives the next (Drew and Abbott, 2003; Li and Greenside, 2006; Jin et al., 2007; Weber and Hahnloser, 2007;
Gibb et al., 2009a, b; Jin, 2009; Long et al., 2010; Amador et al., 2013; Bertram et al., 2014).

Importantly, chain models make the testable predication that damage at any given point along the chain should eliminate production of all syllables downstream from the point of damage. The effects of lateral HVC ablation, where syllables toward the end of the motif are omitted, are entirely consistent with such models. However, the atypical sequencing that follows medial HVC ablation is not consistent with unitary chain model and instead suggests that the sequence of song syllables may be encoded independently within HVC. One possibility is that song may be encoded hierarchically, perhaps similar to Lashley's (1951) model for serial order in behavior (of which birdsong is an example). In Lashley's (1951) model, a superordinate trace coordinates the sequence of behavior and subordinate traces coordinate the structure of each gestural unit. If song is to be encoded in this fashion, a crucial question is how a hierarchical relationship between medial (sequence) and lateral (structure) HVC pathways might be established. The parallel extrinsic connectivity of medial and lateral HVC suggests that this must occur at a downstream site.

\section{How is activity between medial and lateral pathways integrated?}

Our behavioral findings and the anatomical convergence of medial and lateral HVC pathways at RA (the primary motor cortical region for birdsong; Fig. 9A) suggest an integrative function for this region that has not been previously recognized. RA contains inhibitory interneurons and projection neurons that drive brainstem respiratory and vocal-motor regions (Vicario, 1991; Wild, 1993), the thalamus (Goldberg and Fee, 2012), and a small population of RA neurons that are connected reciprocally with HVC (Roberts et al., 2008). Whether HVCRA axons from medial and lateral HVC synapse on the same or different RA neurons is unknown, but the terminals of individual HVCRA axons are known to arborize throughout RA (Mooney, 2000; Yip et al., 2012) where they synapse on inhibitory and projection neurons (Mooney and Konishi, 1991; Mooney, 1992; Spiro et al., 1999; Stark and Perkel, 1999). Thus, HVCRA axons from medial and lateral domains of HVC could converge on the same RA neurons, or they could contact adjacent populations of RA projection neurons and/or interneurons. One possibility is that the medial HVC pathway (sequence) might differentially influence inhibitory neurotransmission within RA, thereby gating the ability of the lateral HVC pathway (structure) to drive the ensembles of RA 
projection neurons that shape the production of individual song syllables (Leonardo and Fee, 2005).

Although individual RA neurons participate in multiple ensembles, a unique pattern of ensemble activity is responsible for each individual syllable, even syllables that share a highly similar acoustic structure (Leonardo and Fee, 2005). Thus, our data from birds with lateral HVC ablations (where individual song syllables were selectively omitted) suggest that syllable-specific populations of HVCRA neurons in lateral HVC are directly linked to the RA ensembles that drive those syllables. Syllable-specific populations of HVCRA neurons in lateral HVC are potentially organized within one or more of the rostral-caudal chains of interconnected neurons that span the medial-lateral axis of HVC (Stauffer et al., 2012). Thus, after lateral HVC ablation, individual RA neurons could still participate in other ensembles, driving syllables that shared acoustic features with the omitted syllables (compare syllables B-D in Fig. 7A).

In conclusion, we have identified a functional topography within HVC: medial and lateral HVC play distinct roles in processing song sequence and structure, respectively. This conclusion is reinforced by our further demonstration that the extrinsic connectivity of medial and lateral HVC is organized in parallel. Interestingly, recent identification of neighboring cortical regions for independent syntactic and lexical processing during human speech production (Menenti et al., 2012) indicates that both humans and zebra finches use a parallel architecture for processing the sequence and structure of learned vocal patterns. Although independent encoding of the sequence and structure of human speech is perhaps self-evident, the prevailing view has been that the sequence and structure of zebra finch song are encoded as a unitary construct. Our demonstration of anatomically distinct pathways that process the sequence and structure of syllables in parallel suggests a surprising convergence in the architecture of vertebrate brain pathways for encoding learned vocal patterns.

\section{References}

Airey DC, DeVoogd TJ (2000) Greater song complexity is associated with augmented song system anatomy in zebra finches. Neuroreport 11:23392344. CrossRef Medline

Amador A, Perl YS, Mindlin GB, Margoliash D (2013) Elemental gesture dynamics are encoded by song premotor cortical neurons. Nature 495 : 59-64. CrossRef Medline

Aronov D, Andalman AS, Fee MS (2008) A specialized forebrain circuit for vocal babbling in the juvenile songbird. Science 320:630-634. CrossRef Medline

Ashmore RC, Bourjaily M, Schmidt MF (2008) Hemispheric coordination is necessary for song production in adult birds: implications for a dual role for forebrain nuclei in vocal motor control. J Neurophysiol 99:373-385. CrossRef Medline

Bertram R, Daou A, Hyson RL, Johnson F, Wu W (2014) Two neural streams, one voice: pathways for theme and variation in the songbird brain. Neuroscience C277:806-817. CrossRef Medline

Bottjer SW, Halsema KA, Brown SA, Miesner EA (1989) Axonal connections of a forebrain nucleus involved with vocal learning in zebra finches. J Comp Neurol 279:312-326. CrossRef Medline

Daou A, Johnson F, Wu W, Bertram R (2012) A computational tool for automated large-scale analysis and measurement of bird-song syntax. J Neurosci Methods 210:147-160. CrossRef Medline

Day NF, Terleski KL, Nykamp DQ, Nick TA (2013) Directed functional connectivity matures with motor learning in a cortical pattern generator. J Neurophysiol 109:913-923. CrossRef Medline

Drew PJ, Abbott LF (2003) Model of song selectivity and sequence generation in area HVc of the songbird. J Neurophysiol 89:2697-2706. CrossRef Medline
Elliott KC, Wu W, Bertram R, Johnson F (2014) Disconnection of a basal ganglia circuit in juvenile songbirds attenuates the spectral differentiation of song syllables. Dev Neurobiol 74:574-590. CrossRef Medline

Fortune ES, Margoliash D (1995) Parallel pathways and convergence onto $\mathrm{HVc}$ and adjacent neostriatum of adult zebra finches (Taeniopygia guttata). J Comp Neurol 360:413-441. CrossRef Medline

Foster EF, Bottjer SW (1998) Axonal connections of the high vocal center and surrounding cortical regions in juvenile and adult male zebra finches. J Comp Neurol 397:118-138. CrossRef Medline

Fujimoto H, Hasegawa T, Watanabe D (2011) Neural coding of syntactic structure in learned vocalizations in the songbird. J Neurosci 31:1002310033. CrossRef Medline

Gerfen CR (2003) Basic neuroanatomical methods. Curr Protoc Neurosci Chapter 1:Unit 1.1. CrossRef Medline

Gibb L, Gentner TQ, Abarbanel HD (2009) Brain stem feedback in a computational model of birdsong sequencing. J Neurophysiol 102:17631778. CrossRef Medline

Gibb L, Gentner TQ, Abarbanel HD (2009) Inhibition and recurrent excitation in a computational model of sparse bursting in song nucleus HVC. J Neurophysiol 102:1748-1762. CrossRef Medline

Goldberg JH, Fee MS (2012) A cortical motor nucleus drives the basal ganglia-recipient thalamus in singing birds. Nat Neurosci 15:620-627. CrossRef Medline

Goldin MA, Alonso LM, Alliende JA, Goller F, Mindlin GB (2013) Temperature induced syllable breaking unveils nonlinearly interacting timescales in birdsong motor pathway. PLoS One 8:e67814. CrossRef Medline

Graber MH, Helmchen F, Hahnloser RH (2013) Activity in a premotor cortical nucleus of zebra finches is locally organized and exhibits auditory selectivity in neurons but not in glia. PLoS One 8:e81177. CrossRef Medline

Hahnloser RH, Kozhevnikov AA, Fee MS (2002) An ultra-sparse code underlies the generation of neural sequences in a songbird. Nature 419:65-70. CrossRef Medline

Hamaguchi K, Tschida KA, Yoon I, Donald BR, Mooney R (2014) Auditory synapses to song premotor neurons are gated off during vocalization in zebra finches. Elife 3:e01833. CrossRef Medline

Jin DZ (2009) Generating variable birdsong syllable sequences with branching chain networks in avian premotor nucleus HVC. Phys Rev E Stat Nonlin Soft Matter Phys 80:051902. CrossRef Medline

Jin DZ, Ramazanoðlu FM, Seung HS (2007) Intrinsic bursting enhances the robustness of a neural network model of sequence generation by avian brain area HVC. J Comput Neurosci 23:283-299. CrossRef Medline

Johnson F, Bottjer SW (1995) Differential estrogen accumulation among populations of projection neurons in the higher vocal center of male canaries. J Neurobiol 26:87-108. CrossRef Medline

Kao MH, Brainard MS (2006) Lesions of an avian basal ganglia circuit prevent context-dependent changes to song variability. J Neurophysiol 96: 1441-1455. CrossRef Medline

Kozhevnikov AA, Fee MS (2007) Singing-related activity of identified HVC neurons in the zebra finch. J Neurophysiol 97:4271-4283. CrossRef Medline

Lashley KS (1951) The problem of serial order in behavior. In: Cerebral mechanisms in behavior (Jeffress LA, ed), pp 112-131. New York: Wiley.

Leonardo A, Fee MS (2005) Ensemble coding of vocal control in birdsong. J Neurosci 25:652-661. CrossRef Medline

Li M, Greenside H (2006) Stable propagation of a burst through a onedimensional homogeneous excitatory chain model of songbird nucleus HVC. Phys Rev E Stat Nonlin Soft Matter Phys 74:011918. CrossRef Medline

Long MA, Fee MS (2008) Using temperature to analyse temporal dynamics in the songbird motor pathway. Nature 456:189-194. CrossRef Medline

Long MA, Jin DZ, Fee MS (2010) Support for a synaptic chain model of neuronal sequence generation. Nature 468:394-399. CrossRef Medline

Luo M, Ding L, Perkel DJ (2001) An avian basal ganglia pathway essential for vocal learning forms a closed topographic loop. J Neurosci 21:68366845. Medline

MacDougall-Shackleton SA, Hulse SH, Ball GF (1998) Neural correlates of singing behavior in male zebra finches (Taeniopygia guttata). J Neurobiol 36:421-430. CrossRef Medline

Margoliash D, Fortune ES, Sutter ML, Yu AC, Wren-Hardin BD, Dave A (1994) Distributed representation in the song system of oscines: evolu- 
tionary implications and functional consequences. Brain Behav Evol 44: 247-264. CrossRef Medline

Menenti L, Segaert K, Hagoort P (2012) The neuronal infrastructure of speaking. Brain Lang 659 122:71-80. CrossRef Medline

Mooney R (1992) Synaptic basis for developmental plasticity in a birdsong nucleus. J Neurosci 12:2464-2477. Medline

Mooney R (2000) Different subthreshold mechanisms underlie song selectivity in identified HVc neurons of the zebra finch. J Neurosci 20:54205436. Medline

Mooney R, Konishi M (1991) Two distinct inputs to an avian song nucleus activate different glutamate receptor subtypes on individual neurons. Proc Natl Acad Sci U S A 88:4075-4079. CrossRef Medline

Olson CR, Rodrigues PV, Jeong JK, Prahl DJ, Mello CV (2011) Organization and development of zebra finch HVC and paraHVC based on expression of zRalDH, an enzyme associated with retinoic acid production. J Comp Neurol 519:148-161. CrossRef Medline

Poole B, Markowitz JE, Gardner TJ (2012) The song must go on: resilience of the songbird vocal motor pathway. PLoS One 7:e38173. CrossRef Medline

Roberts TF, Klein ME, Kubke MF, Wild JM, Mooney R (2008) Telencephalic neurons monosynaptically link brainstem and forebrain premotor networks necessary for song. J Neurosci 28:3479-3489. CrossRef Medline

Spiro JE, Dalva MB, Mooney R (1999) Long-range inhibition within the zebra finch song nucleus RA can coordinate the firing of multiple projection neurons. J Neurophysiol 81:3007-3020. Medline

Stark LL, Perkel DJ (1999) Two-stage, input-specific synaptic maturation in a nucleus essential for vocal production in the zebra finch. J Neurosci 19:9107-9116. Medline

Stauffer TR, Elliott KC, Ross MT, Basista MJ, Hyson RL, Johnson F (2012) Axial organization of a brain region that sequences a learned pattern of behavior. J Neurosci 32:9312-9322. CrossRef Medline

Sutter ML, Margoliash D (1994) Global synchronous response to autogenous song in zebra finch HVc. J Neurophysiol 72:2105-2123. Medline

Tchernichovski O, Nottebohm F, Ho CE, Pesaran B, Mitra PP (2000) A procedure for an automated measurement of song similarity. Anim Behav 59:1167-1176. CrossRef Medline

Thompson JA, Johnson F (2007) HVC microlesions do not destabilize the vocal patterns of adult male zebra finches with prior ablation of LMAN. Dev Neurobiol 67:205-218. CrossRef Medline

Thompson JA, Wu W, Bertram R, Johnson F (2007) Auditory-dependent vocal recovery in adult male zebra finches is facilitated by lesion of a forebrain pathway that includes the basal ganglia. J Neurosci 27:12308 12320. CrossRef Medline

Thompson JA, Basista MJ, Wu W, Bertram R, Johnson F (2011) Dual premotor contribution to songbird syllable variation. J Neurosci 31:322-330. CrossRef Medline

Vicario DS (1991) Neural mechanisms of vocal production in songbirds. Curr Opin Neurobiol 1:595-600. CrossRef Medline

Wang CZ, Herbst JA, Keller GB, Hahnloser RH (2008) Rapid interhemispheric switching during vocal production in a songbird. PLoS Biol 6:e250. CrossRef Medline

Weber AP, Hahnloser RH (2007) Spike correlations in a songbird agree with a simple Markov population model. PLoS Comput Biol 3:e249. CrossRef Medline

Wild JM (1993) Descending projections of the songbird nucleus robustus archistriatalis. J Comp Neurol 338:225-241. Medline

Wild JM, Williams MN, Howie GJ, Mooney R (2005) Calcium-binding proteins define interneurons in HVC of the zebra finch (Taeniopygia guttata). J Comp Neurol 483:76-90. CrossRef Medline

Wu W, Thompson JA, Bertram R, Johnson F (2008) A statistical method for quantifying songbird phonology and syntax. J Neurosci Methods 174: 147-154. CrossRef Medline

Yip ZC, Miller-Sims VC, Bottjer SW (2012) Morphology of axonal projections from the high vocal center to vocal motor cortex in songbirds. J Comp Neurol 520:2742-2756. CrossRef Medline

Yu AC, Margoliash D (1996) Temporal hierarchical control of singing in birds. 273:1871-1875. 